\title{
Ligne directrice sur le dépistage de l'adénocarcinome œsophagien chez les patients atteints de reflux gastro-œsophagien chronique
}

\author{
Stéphane Groulx MD, Heather Limburg MSc, Marion Doull PhD, Scott Klarenbach MD MSc, Harminder Singh MD MPH, \\ Brenda J. Wilson MBChB MSc MRCP, Brett Thombs PhD; pour le Groupe d'étude canadien sur les soins de santé préventifs
}

Citation : CMAJ 2020 July 6;192:E768-77. doi: 10.1503/cmaj.190814-f

Entrevue avec l'auteur en baladodiffusion : https://soundcloud.com/cmajpodcasts/190814-guide-fre

Voir le commentaire connexe (en anglais) au www.cmaj.ca/lookup/doi/10.1503/cmaj.200697

$\mathbf{E}$ n 2019, on a estimé à 6 par 100000 le nombre de nouveaux cas de cancer de l'œsophage diagnostiqués chez les Canadiens ${ }^{1}$. Évalué à $15 \%$, le taux de survie net à 5 ans est parmi les plus faibles de tous les pronostics de cancer ${ }^{1}$. Les hommes présentent une incidence plus élevée que les femmes, avec environ 9 cas contre 2 par 100000 , respectivement ${ }^{1}$. L'adénocarcinome est le type le plus fréquent de cancer de l'œsophage au Canada, suivi du carcinome épidermoïde ${ }^{2}$. L'incidence s'est modifiée depuis une quarantaine d'années, avec des taux d'adénocarcinome en hausse et des taux de carcinome épidermoïde en baisse (figure 1$)^{3}$. Ce changement peut être dû à une augmentation des facteurs de risque d'adénocarcinome (p. ex., reflux gastro-œsophagien, obésité) et à une diminution des facteurs de risque de carcinome épidermoïde (p. ex., tabagisme) $)^{2}$. Cette ligne directrice se concentre donc sur le dépistage de l'adénocarcinome œsophagien.

Les plus importants facteurs de risque pour l'adénocarcinome œsophagien sont : certaines conditions précancéreuses (p. ex., œsophage de Barrett, dysplasie œsophagienne), âge plus avancé ( $\geq 50$ ans), reflux gastro-œsophagien (RGO), sexe masculin, antécédents familiaux, race ou ethnicité blanche, obésité abdominale et tabagisme ${ }^{4-10}$.

Le reflux gastro-œsophagien (définition internationale de Montréal ${ }^{11}$ ) est un problème répandu, caractérisé par une remontée du contenu gastrique (régurgitation acide) qui cause des symptômes désagréables (p. ex., brûlures d'estomac, aigreurs). Il faut distinguer le RGO de la dyspepsie, qui est un syndrome principalement caractérisé par une douleur épigastrique d'une durée d'au moins 1 mois ${ }^{12}$. En Amérique du Nord et en Europe, la prévalence du RGO (basée sur une manifestation hebdomadaire des symptômes) toucherait de $10 \%$ à $20 \%$ de la population selon les estimations ${ }^{13,14}$.

Le reflux gastro-œsophagien multiplie par 5 à 7 la probabilité de souffrir d'un adénocarcinome œsophagien (35 cas contre 7 par 100000 hommes à l'âge de 60 ans); $60 \%$ des patients atteints

\section{POINTS CLÉS}

- Le Groupe d'étude canadien sur les soins de santé préventifs recommande de ne pas dépister les adultes atteints de reflux gastro-œsophagien (RGO) chronique pour l'adénocarcinome œsophagien et ses précurseurs (œsophage de Barrett ou dysplasie), parce que le dépistage n'apporte aucun bénéfice probant, qu'il implique des préjudices incertains, une mobilisation importante des ressources, et que les valeurs et préférences des patients face à ce dépistage sont variables.

- Une seule étude de cohorte rétrospective (très faible certitude des données probantes) a révélé que le dépistage chez les patients atteints de RGO chronique a identifié un plus grand nombre de cas d'adénocarcinome œsophagien à un stade précoce, mais n'a mis en évidence aucune différence sur le plan de la survie à long terme (mortalité de toute cause).

- L'acceptabilité du dépistage a été variable en raison des valeurs et préférences individuelles; certaines personnes tenaient au dépistage en raison de facteurs de risque individuels et familiaux, de convictions personnelles ou d'une crainte de passer à côté d'un diagnostic précoce, tandis que d'autres s'inquiétaient du caractère invasif du dépistage et des risques associés.

- Cette ligne directrice sur le dépistage ne s'applique pas aux personnes qui manifestent des symptômes d'alarme susceptibles d'être causés par l'adénocarcinome œsophagien (p. ex., dysphagie, odynophagie, vomissements récurrents, perte de poids inexpliquée, anémie, inappétence ou saignement gastro-intestinal) ni aux personnes qui ont un diagnostic d'œsophage de Barrett (avec ou sans dysplasie).

de ce cancer disent avoir souffert de RGO ${ }^{15-17}$. Par contre, la majorité des gens qui souffrent de RGO chronique ne développent pas d'adénocarcinome de l'œsophage, et il est toujours difficile de prédire la progression ${ }^{18}$. Un modèle de Markov appliqué à des personnes de race blanche, non hispaniques, âgées de 60 ans qui présentaient des symptômes de RGO au moins toutes 


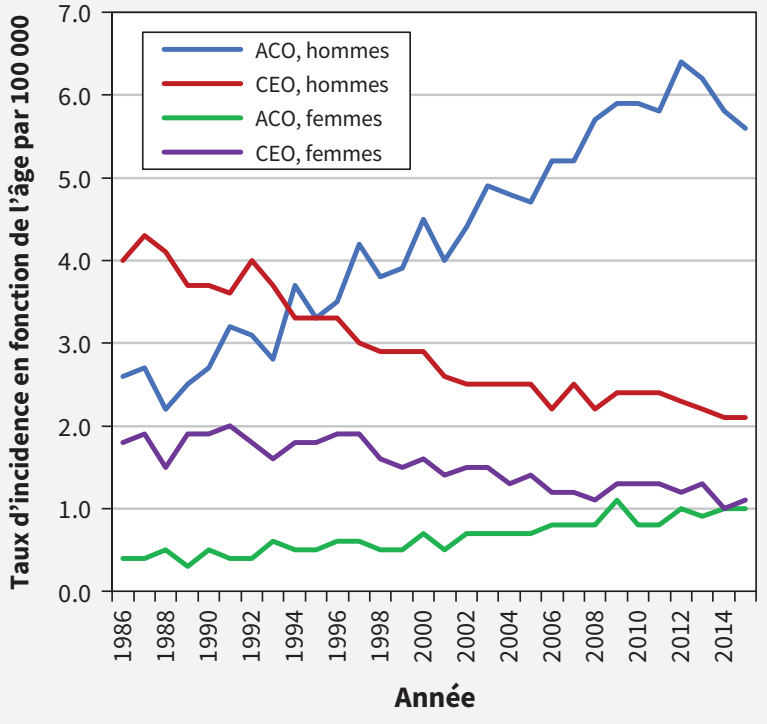

Figure 1 : Taux d'incidence selon l'âge* de l'adénocarcinome œsophagien (ACO) et du carcinome épidermoïde œsophagien (CEO), tous âges, Canada (sauf le Québec)†, de 1986 à 2015. Source des données: Bases de données du Registre canadien du cancer et du Système national de déclaration des cas de cancer de Statistique Canada. Analysé par : le Centre de surveillance et de recherche appliquée de l'Agence de la santé publique du Canada. *Standardisé selon l'âge pour la population canadienne de 2011; voir annexe $1 \mathrm{~F}$ pour les codes topographiques et morphologiques du cancer œsophagien inclus. †Les données pour le Québec ont été exclues parce qu'elles n'étaient pas disponibles pour toutes les périodes.

les semaines a estimé l'incidence de l'adénocarcinome œsophagien à 35 par 100000 années-personnes chez les hommes et à 4 par 100000 années-personnes chez les femmes ${ }^{17}$.

L'œsophage de Barrett est une adaptation métaplasique des cellules de l'œsophage où une muqueuse de type intestinal remplace la muqueuse squameuse normale ${ }^{19,20}$. Environ de $5 \%$ à $15 \%$ des patients atteints de RGO développent un œsophage de Barrett, contre $1 \%$ à $2 \%$ de la population générale ${ }^{10}$. La dysplasie peut se manifester de manière indépendante, mais elle est plus fréquente chez les gens qui ont un œsophage de Barrett ${ }^{21}$. On croit que l'histoire naturelle de l'adénocarcinome œsophagien suit une progression séquentielle allant du RGO à l'œsophage de Barrett, vers une dysplasie de bas grade, puis de haut grade ${ }^{5,22,23}$. Le risque de souffrir d'un adénocarcinome œsophagien consécutif à un œsophage de Barrett augmente d'un niveau de 0,3\% par année en l'absence de dysplasie, à $0,5 \%$ en présence de dysplasie de bas grade et à $6 \%$ en présence de dysplasie de haut grade ${ }^{24-26}$.

Environ $93 \%$ des diagnostics d'adénocarcinome œesophagien sont posés lors de l'investigation de patients qui présentent des symptômes d'alarme, tels dysphagie, odynophagie, vomissements récurrents, perte de poids inexpliquée, anémie, inappétence ou saignements gastro-intestinaux, qui peuvent être associés à un adénocarcinome de stade avancé $e^{7,27-29}$.

Le Groupe d'étude canadien sur les soins de santé préventifs a voulu savoir si le dépistage endoscopique des patients atteints de RGO chronique sans symptômes d'alarme permettait de dépister le cancer à un stade plus précoce, d'identifier des conditions précancéreuses traitables (œsophage de Barrett, dysplasie), de réduire la progression vers un adénocarcinome œsophagien ou de réduire la mortalité.

\section{Portée}

Cette ligne directrice s'adresse aux professionnels en soins primaires, c'est-à-dire, aux professionnels de la santé qui offrent l'accès à des soins complets et coordonnés sur une base continue, et représentent le premier point de contact avec le système de santé ${ }^{30}$. La population cible se compose d'adultes atteints de RGO chronique et exclut ceux qui ont des symptômes d'alarme ou qui ont déjà un diagnostic d'œsophage de Barrett (avec ou sans dysplasie).

Le Groupe d'étude canadien a initialement défini le RGO chronique comme suit : symptômes de RGO d'une durée de 12 mois ou plus (sans fréquence spécifique) ou utilisation d'un inhibiteur de la pompe à protons (ou autre pharmacothérapie) pour traiter le RGO d'une durée de 12 mois ou plus ${ }^{31,32}$. Toutefois, nous avons ensuite élargi la définition afin d'inclure dans la revue des données probantes pour la ligne directrice toute étude sur le « RGO chronique » basée sur les critères des auteurs des études ${ }^{33-35}$.

\section{Recommandation}

Nous recommandons de ne pas faire de dépistage de l'adénocarcinome œsophagien ou de ses précurseurs (œsophage de Barrett ou dysplasie) chez les adultes ( $\geq 18$ ans) atteints de RGO chronique (forte recommandation; très faible certitude des données probantes).

Cette recommandation ne s'applique pas aux personnes qui manifestent des symptômes d'alarme ou qui ont déjà reçu un diagnostic d'œsophage de Barrett (avec ou sans dysplasie).

Même si des facteurs de risque tels que âge ( $\geq 50$ ans), sexe masculin, antécédents familiaux, race ou ethnicité blanche, obésité abdominale et tabagisme peuvent accroître le risque d'adénocarcinome œsophagien, les essais et études de cohorte pertinents n'incluaient pas suffisamment de données de chacune de ces catégories pour appuyer une modification de notre recommandation quant à un dépistage en fonction de ces facteurs, pris ensemble ou séparément (annexe 1C, accessible en anglais ici : www.cmaj.ca/ lookup/suppl/doi:10.1503/cmaj.190814/-/DC2.33,35. Voir encadré no 1 pour les définitions utilisées dans les lignes directrices du Groupe d'étude canadien concernant les degrés de certitude des données probantes et la force des recommandations $\mathrm{s}^{36}$.

\section{Dépistage}

Une revue systématique a recensé 2 études de cohorte rétrospectives basées sur des dossiers médicaux électroniques qui ont évalué l'efficacité (bénéfices et préjudices) du dépistage par rapport à l'absence de dépistage chez les patients atteints de RGO chronique ${ }^{39,40}$. La gravité ou la durée du RGO n'étaient pas définies dans ces études. L'étude de Rubenstein et ses collaborateurs 
portait sur 155 patients porteurs d'un diagnostic d'adénocarcinome œsophagien et ayant subi $(n=25)$ ou non $(n=130)$ une œsophagogastroduodénoscopie au cours des 5 années précédentes. Pour ce qui est des bénéfices potentiels, cette étude n'a révélé aucune amélioration statistiquement significative de la survie à long terme (mortalité de toute cause) (rapport de risque $[R R]$ ajusté, 0,93, intervalle de confiance $[I C]$ de $95 \%, 0,58$ à 1,50) (très faible certitude des données probantes) ${ }^{39}$. La même étude a révélé un effet absolu statistiquement significatif chez les patients qui avaient subi une oesophagogastroduodénoscopie : 156 de plus par 1000 (IC de $95 \%$, 5 à 486 de plus par 1000) (très faible certitude des données probantes) ont reçu un diagnostic d'adénocarcinome œsophagien de stade moins avancé (stade $1 \mathrm{c}$. stades 2-4) (tableau 1). La deuxième étude de cohorte rétrospective disposait de données insuffisantes pour déterminer si le dépistage permettait un diagnostic à un stade moins avancé ou une baisse de la mortalité ${ }^{40}$. Les 2 études présentaient des données insuffisantes pour procéder à des analyses de sous-groupes en fonction des facteurs de risque (annexe 1C) ${ }^{33,35}$. Aucune des études incluses n'a rapporté ou fourni de données concernant les autres issues cliniques d'intérêt identifiées (c.-à-d., mortalité par cause spécifique, qualité de vie, interventions médicales supplémentaires ou surdiagnostic) ${ }^{33,35}$. Cinq essais randomisés et contrôlés ${ }^{41-45}$ (ERC) et 1 étude de cohorte $^{46}$ ont comparé différentes modalités de dépistage de l'œsophage de Barrett, dont l'œsophagogastroduodénoscopie sous sédation, l'œsophagoscopie transnasale sans sédation, l'œsophagoscopie par vidéocapsule (avalée) et l'œsophagoscopie transorale sans sédation ${ }^{33,35}$. Lorsqu'elle était rapportée, la définition du RGO variait d'une étude à l'autre; certaines études n'ont pas mentionné la durée du $\mathrm{RGO}^{42,45,46}$ ou l'utilisation des inhibiteurs de la pompe de protons ${ }^{42,45}$; et aucune n'a utilisé la définition de Montréal ${ }^{11}$. On n'a noté aucune différence statistiquement significative quant aux taux de dépistage de l'œsophage de Barrett confirmé ou de la dysplasie entre les différentes modalités, et aucun cas d'adénocarcinome œsophagien n'a été dépisté (très faible certitude des données probantes) $)^{33,35}$.

\section{Encadré no 1 : Classification des recommandations ${ }^{36}$}

Les recommandations sont classifiées selon le système GRADE (Grading of Recommendations Assessment, Development and Evaluation system $)^{37}$. Qu'une recommandation soit forte ou conditionnelle* dépend de facteurs tels que la certitude des effets d'une intervention, y compris leur ampleur, de même que des estimations de la façon dont les patients évaluent et priorisent les résultats, de la variabilité de ces estimations et d'une utilisation judicieuse des ressources.

\section{Recommandations fortes}

Les recommandations fortes sont celles à propos desquelles le Groupe d'étude canadien sur les soins de santé préventifs considère que les effets escomptés d'une intervention en surpassent les conséquences indésirables (recommandation forte à l'appui de l'intervention) ou que les conséquences indésirables d'une intervention en surpassent les effets escomptés (recommandation forte à l'encontre de l'intervention). Une recommandation forte suppose que les intérêts de la plupart des individus seront mieux servis par la mesure recommandée.

Les recommandations fortes se fondent normalement sur des données probantes de grande certitude (c.-à-d., grande fiabilité de l'effet estimé d'une intervention). Les recommandations fortes peuvent préconiser une intervention (degré de confiance élevé quant aux bénéfices escomptés) ou la déconseiller (degré de confiance élevé quant à la probabilité de préjudices). Toutefois, il y a des circonstances où une forte recommandation peut être envisagée en fonction de données probantes de faible ou de très faible certitude, ou en l'absence de données probantes ou si elles sont de faible certitude quant à un bénéfice.

Lorsqu'il y a absence de données probantes pour croire qu'il y a un bénéfice à appliquer un nouveau service de prévention ou lorsqu'une conclusion quant à un bénéfice possible requiert un degré élevé de spéculation quant aux liens avec des données de faible certitude, mais qu'il y a une forte certitude que certains patients pourraient subir un préjudice ou que les ressources précieuses du système de santé pourraient être utilisées à mauvais escient, le Groupe d'étude canadien peut formuler une forte recommandation à l'encontre de l'application dudit service. Ceci concorde avec l'approche $\mathrm{GRADE}^{38}$, selon laquelle de fortes recommandations sont parfois formulées sur la base de preuves de faible certitude, alliées à une forte certitude quant à des préjudices ou à une utilisation indue des ressources, et compte tenu de la valeur qu'accorde le Groupe d'étude canadien à l'utilisation judicieuse des ressources en soins primaires.

\section{Recommandations conditionnelles}

Les recommandations conditionnelles sont celles à propos desquelles les effets escomptés surpassent probablement les conséquences indésirables (recommandation conditionnelle en faveur d'une intervention) ou les conséquences indésirables surpassent probablement les effets escomptés (recommandation conditionnelle à l'encontre d'une intervention), mais une incertitude marquée existe. Les recommandations conditionnelles sont formulées lorsque la certitude des données probantes est plus faible, lorsque la frontière entre effets recherchés et conséquences indésirables est mince et lorsque l'équilibre dépend des valeurs et préférences des patients, ou lorsqu'il y a une forte variabilité entre les valeurs et les préférences des patients. Les cas où le rapport coût:bénéfice est ambigu, où les parties prenantes clés ne s'entendent pas sur l'acceptabilité ou la faisabilité de l'intervention, et où l'effet sur l'équité en matière de santé est indéterminé, conduiront probablement à une recommandation conditionnelle.

Dans certains cas où on formule une recommandation conditionnelle concernant une intervention, les cliniciens sont encouragés à impliquer leurs patients dans un processus de prise de décision partagée, à reconnaître que différents choix seront appropriés selon les patients et à aider chacun d'entre eux à prendre une décision relative à une intervention qui est en accord avec ses valeurs et ses préférences. Cela exige des cliniciens qu'ils reconnaissent que différents choix peuvent être appropriés selon les patients et que ces décisions doivent concorder avec les valeurs et préférences de ces derniers. Des outils d'application des connaissances sont disponibles sur le site Web du Groupe d'étude canadien (www.canadiantaskforce.ca) pour promouvoir une prise de décision fondée sur des données probantes et en accord avec les priorités des individus.

Les données probantes sont jugées de certitude forte, modérée, faible ou très faible, selon la probabilité que des recherches plus approfondies modifient le degré de confiance du Groupe d'étude canadien à l'endroit de l'effet attendu de l'intervention.

*Le Groupe d'étude canadien utilisait autrefois le terme « recommandation faible », mais l'a remplacé par le terme «recommandation conditionnelle » pour plus de clarté et pour faciliter l'application des recommandations, en s'inspirant des commentaires formulés par les médecins utilisateurs des connaissances. Ce changement a notamment été motivé par l'importance que le Groupe d'étude canadien accorde au processus de prise de décision partagée et à la nécessité de préciser si l'application d'une recommandation dépend de certaines circonstances, telles que les valeurs des patients, la disponibilité des ressources ou d'autres facteurs contextuels. 
Tableau 1 : sommaire des issues cliniques cernant le dépistage c. l'absence de dépistage de l'adénocarcinome œsophagien chez les personnes atteintes de reflux gastro-œsophagien chronique

\begin{tabular}{|c|c|c|c|c|c|c|c|}
\hline $\begin{array}{l}\text { Issues } \\
\text { cliniques }\end{array}$ & $\begin{array}{l}\mathrm{N}^{\text {bre }} \text { et type } \\
\text { d'étude } \\
\text { (référence) }\end{array}$ & Dépistage & $\begin{array}{l}\text { Absence de } \\
\text { dépistage }\end{array}$ & $\begin{array}{l}\text { Risque relatif } \\
\text { (IC de } 95 \% \text { ) }\end{array}$ & $\begin{array}{l}\text { Différence absolue } \\
\text { par } 1000 \\
\text { (IC de } 95 \%)\end{array}$ & $\begin{array}{l}\text { Augmentation } \\
\text { du risque } \\
\text { absolu \% }\end{array}$ & $\begin{array}{l}\text { Certitude } \\
\text { des données } \\
\text { probantes }\end{array}$ \\
\hline Survie & $\begin{array}{l}1 \text { étude de } \\
\text { cohorte } \\
\text { rétrospective } \\
\text { (Rubenstein et } \\
\text { coll., 2008) }\end{array}$ & \multicolumn{5}{|c|}{$\begin{array}{l}\text { Résumé narratif : Les auteurs de l'étude ont signalé qu'il n'y avait pas de différence de survie à } \\
\text { long terme entre les sujets selon qu'ils avaient ou non déjà subi une OGD (HR 0,82, IC de } 95 \% \\
0,52-1,29) \text {. Les ajustements pour tenir compte de l'âge, des comorbidités et du nombre } \\
\text { d'années depuis le diagnostic ont généré des résultats similaires (HR 0,93, IC de } 95 \% 0,58-1,50 \text { ). }\end{array}$} & Très faible \\
\hline \multirow[t]{2}{*}{$\begin{array}{l}\text { Stade } 1 \text { au } \\
\text { moment du } \\
\text { diagnostic }\end{array}$} & $\begin{array}{l}1 \text { étude de } \\
\text { cohorte } \\
\text { rétrospective } \\
\text { (Rubenstein et } \\
\text { coll., 2008) }\end{array}$ & $7 / 25$ & $16 / 130$ & $\begin{array}{c}2,27 \\
(1,04 \text { à } 4,95)\end{array}$ & $\begin{array}{l}156 \text { par } 1000 \text { de } \\
\text { plus avaient un } \\
\text { stade moins avancé } \\
\text { (stade } 1 \text { c. stades } \\
2-4 \text { ) au moment du } \\
\text { diagnostic s'ils } \\
\text { avaient déjà subi } \\
\text { une OGD } \\
\text { (5 de plus à } 486 \text { de } \\
\text { plus) }\end{array}$ & 15,7 & Très faible* \\
\hline & $\begin{array}{l}1 \text { étude de } \\
\text { cohorte } \\
\text { rétrospective } \\
\text { (Hammad et } \\
\text { coll., 2019) }\end{array}$ & \multicolumn{5}{|c|}{$\begin{array}{l}\text { Résumé narratif : } 1 \text { patient sur } 153 \text {, non surveillé pour œsophage de Barrett, avait subi une } \\
\text { OGD au cours des } 5 \text { années précédentes; } 15 \text { autres avaient subi une OGD }>5 \text { ans auparavant, } \\
\text { sans détails supplémentaires sur le moment. Aux fins de cette revue, ces patients ont été inclus } \\
\text { avec ceux qui n'avaient jamais subi d'OGD. Le patient ayant subi le dépistage a reçu un } \\
\text { diagnostic d'adénocarcinome œsophagien de stade incertain. }\end{array}$} & Très faible* \\
\hline
\end{tabular}

Quatre ERC ont évalué les préjudices causés par le

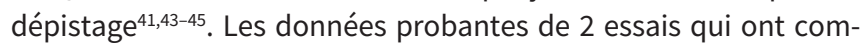
paré l'œsophagogastroduodénoscopie sous sédation à l'œsophagoscopie transnasale sans sédation $(n=209)^{43}$, et l'intervention transnasale sans sédation à l'œsophagogastroduodénoscopie transorale sans sédation $(n=59)^{45}$ ont fait état d'un événement indésirable grave (après l'endoscopie transnasale) $)^{33,35}$. Dans 3 ERC, l'œsophagoscopie transnasale sans sédation a été associée à une augmentation statistiquement significative de l'anxiété (préjudice) comparativement à l'œsophagogastroduodénoscopie sous sédation (durant l'intervention) ou l'œsophagoscopie par vidéocapsule (avant et durant l'intervention) (très faible certitude des données probantes) ${ }^{41,43,44}$ (tableau 2). Toutefois, le léger inconfort additionnel a semblé bien toléré, étant donné que $70 \%$ à $95 \%$ des participants ont déclaré qu'ils se soumettraient de nouveau à l'intervention ${ }^{41-45}$.

\section{Traitement}

Étant donné le peu de données probantes directes disponibles sur l'efficacité du dépistage, le Groupe d'étude canadien a aussi analysé les données probantes indirectes sur l'efficacité du traitement de l'œsophage de Barrett, de la dysplasie ou de l'adénocarcinome œsophagien de stade ${ }^{35,47}$. Cette analyse s'est attardée aux approches thérapeutiques endoscopiques, qui sont plus pertinentes pour les cancers découverts lors d'un dépistage (c.-à-d., à un stade précoce); cependant, l'œsophagectomie demeure le traitement standard pour le cancer de l'œsophage localisé qui a progressé au-delà des tout premiers stades ${ }^{5,48}$.
Pour ce qui est des bénéfices potentiels des traitements, les résultats montrent que le traitement photodynamique, l'ablation par radiofréquence et la résection muqueuse endoscopique de l'œsophage de Barrett (avec ou sans inhibiteurs de la pompe de protons) donnent lieu à une augmentation statistiquement significative de l'éradication ou de l'élimination de la dysplasie (très faible à faible certitude des données probantes) (annexe 1D) 35,47 . Une possible réduction de la progression vers l'adénocarcinome œsophagien a aussi été observée avec le traitement photodynamique (très faible à faible certitude des données probantes) ${ }^{35,47}$. Les résultats concernant la mortalité étaient très limités (taux d'événements de 0 à 3 par essai) ${ }^{35,47}$. Pour l'ensemble des issues cliniques, des données probantes de faible à très faible certitude soutiennent que ces traitements améliorent l'éradication ou l'élimination de la dysplasie, mais leur bénéfice au plan de la mortalité est inconnu.

Une évaluation des complications des traitements a révélé une augmentation statistiquement significative des sténoses et des rétrécissements de l'œsophage associée à la résection muqueuse endoscopique comparativement à l'ablation par radiofréquence (très faible certitude des données probantes) (annexe 1E) ${ }^{35,47}$. Comparativement à l'oméprazole seul, le traitement photodynamique plus oméprazole a donné lieu à une augmentation statistiquement significative de la formation de sténoses (très faible certitude des données probantes ${ }^{35,47}$. Aucune donnée n'a porté sur les autres issues cliniques d'intérêt identifiées (qualité de vie, effets psychologiques des traitements, interventions médicales supplémentaires à la suite du traitement ou surdiagnostic) $)^{35,47}$. 
Tableau 2 : Sommaire des issues cliniques concernant les préjudices avant et durant le dépistage de l'adénocarcinome

œsophagien chez les personnes atteintes de reflux gastro-œesophagien chronique (selon la modalité de dépistage)

\begin{tabular}{|c|c|c|c|c|c|c|c|}
\hline Issue clinique & $\begin{array}{l}\mathrm{N}^{\text {bre }} \text { et type } \\
\text { d'étude } \\
\text { (référence) }\end{array}$ & \multicolumn{2}{|c|}{ Modalités de dépistage } & $\begin{array}{c}\text { Risque } \\
\text { relatif } \\
\text { (IC de } 95 \%)\end{array}$ & $\begin{array}{c}\text { Différence absolue } \\
\text { par } 1000 \\
\text { (IC de } 95 \%)\end{array}$ & $\begin{array}{l}\text { Augmentation } \\
\text { du risque } \\
\text { absolu } \%\end{array}$ & $\begin{array}{l}\text { Certitude } \\
\text { des } \\
\text { données } \\
\text { probantes }\end{array}$ \\
\hline \multirow{2}{*}{$\begin{array}{l}\text { Conséquences } \\
\text { menaçant le } \\
\text { pronostic vital, } \\
\text { graves ou } \\
\text { médicalement } \\
\text { significatives }\end{array}$} & $\begin{array}{l}1 \text { ERC (Sami et } \\
\text { coll., 2015) }\end{array}$ & $\begin{array}{l}\text { OGD standard } \\
\text { sous sédation : } \\
0 / 61\end{array}$ & $\begin{array}{l}\text { OTN sans sédation }{ }^{\star}: \\
0 / 148\end{array}$ & \multicolumn{3}{|c|}{$\begin{array}{l}\text { Résumé narratif : non évaluable, en raison du nombre } 0 \\
\text { dans les } 2 \text { groupes (les événements indésirables graves } \\
\text { ont été évalués aux jours } 1 \text { et } 30 \text { suivant l'intervention). }\end{array}$} & Très faible† \\
\hline & $\begin{array}{l}1 \text { ERC (Zaman } \\
\text { et coll., 1999) }\end{array}$ & $\begin{array}{l}\text { OGD transorale } \\
\text { sans sédation: } \\
0 / 34\end{array}$ & $\begin{array}{l}\text { OTN sans sédation : } \\
1 / 25\end{array}$ & \multicolumn{3}{|c|}{$\begin{array}{l}\text { Résumé narratif : Non évaluable, en raison du nombre } 0 \\
\text { dans le groupe de comparaison. }\end{array}$} & Très faiblet \\
\hline $\begin{array}{l}\text { Anxiété avant } \\
\text { l'intervention }\end{array}$ & $\begin{array}{c}1 \text { ERC } \\
\text { (Chak et coll., } \\
2014)^{41}\end{array}$ & $\begin{array}{l}\text { OTN sans sédation: } \\
33 / 87\end{array}$ & $\begin{array}{l}\text { OVC (dispositif } \\
\text { avalé) : } 15 / 90\end{array}$ & $\begin{array}{c}2,28 \\
(1,33 \text { à } 3,88)\end{array}$ & $\begin{array}{l}213 \text { par } 1000 \text { de plus } \\
\text { ont éprouvé de } \\
\text { l'anxiété avec l'OTN } \\
\text { sans sédation } \\
\text { ( } 55 \text { de plus à } 480 \text { de } \\
\text { plus) }\end{array}$ & 21,3 & Très faible $†$ \\
\hline
\end{tabular}

1 ERC OGD standard

(Jobe et coll., sous sédation :

2006) $)^{44} \quad$ Aucune : $64 / 116$

Légère : 26/116

Modérée : 16/116

Intense : $10 / 116$

1 ERC

(Zaman et

coll., 1999) ${ }^{45}$

OTN sans sédation :

Moyenne $\pm \mathrm{SE}$

$3,0 \pm 0,6$

$1 \mathrm{ERC}$

Anxiété durant

l'insertion

Anxiété durant

l'intervention

1 ERC

(Zaman et coll., 1999) ${ }^{45}$

$1 \mathrm{ERC}$
OTN sans sédation :

Aucune : 59/116

Légère : 39/116

Modérée : $13 / 116$

Intense : 5/116

OGD transorale sans sédation : Moyenne \pm SE 3,0 $\pm 0,5$

OTN sans sédation : Aucune : 45/116 Légère : $43 / 116$

Modérée : 19/116

Intense : 9/116

(Chak et coll.

2014) $)^{41}$

$\begin{array}{cl}1 \text { ERC } & \text { OGD standard } \\ 2015)^{43} & \text { sous sédation: } \\ & 0,8 \pm 1,5 \\ & (40)(40)\end{array}$

1 ERC OGD standard

(Jobe et coll., sous sédation

2006) ${ }^{44} \quad$ Aucune : $87 / 116$

Légère : $11 / 116$

Modérée : $11 / 116$

Intense : $7 / 116$

1 ERC

(Zaman et

OTN sans sédation :

Moyenne \pm SE

coll., 1999) $)^{45}$

$3,3 \pm 0,7$
OGD transorale sans

sédation :

Moyenne $\pm \mathrm{SE}$

$4,7 \pm 0,5$

OVC (dispositif

avalé) : 14/90

OTN sans sédation :

Moyenne \pm SD

OTN à l'hôpital :

$2,3 \pm 2,2$

OTN en clinique

ambulatoire :

$2,8 \pm 2,8$

OTN sans sédation :

Aucune : 62/116

Légère : $38 / 116$

Modérée : 12/116

Intense : 4/116

OGD transorale sans

sédation :

Moyenne $\pm \mathrm{SE}$

$3,3 \pm 0,5$
Résumé narratif: Les auteurs ont fait état des individus ayant éprouvé une anxiété nulle et bénigne, modérée et grave. Aucune différence entre les modalités de dépistage $(p=0,08)$

Résumé narratif : Les auteurs ont fait état de la moyenne pour l'anxiété avant l'intervention. Aucune différence statistiquement significative entre les modalités de dépistage $(p=0,39)$.

Résumé narratif: Les auteurs ont fait état des individus n'ayant éprouvé aucune anxiété ou une anxiété légère, modérée et sévère. Différence statistiquement significative entre les modalités $(p<0,01)$, les sujets assignés à l'OTN sans sédation ayant manifesté plus d'anxiété durant l'insertion.

Résumé narratif : Les auteurs ont fait état de la moyenne pour l'anxiété pendant l'insertion. On n'a noté aucune différence statistiquement significative entre les modalités de dépistage $(p=0,63)$.

\begin{tabular}{cl}
\multicolumn{1}{c}{2,14} & 177 par 1000 de plus \\
$(1,22$ à 3,77$)$ & ont éprouvé de \\
& l'anxiété avec l'OTN \\
& sans sédation (34 de \\
& plus à 431 de plus)
\end{tabular}

Résumé narratif : Les auteurs ont fait état des résultats en utilisant des moyennes sur une échelle de 0 à 10 , où 10 correspondait à grave. Différences statistiquement significatives entre les modalités, les individus assignés à l'OTN sans sédation ayant manifesté plus d'anxiété durant l'intervention $(p<0,01)$.

Résumé narratif : Les auteurs ont fait état des résultats en utilisant le nombre de participants ayant qualifié leur anxiété comme suit « aucune », " légère », « modérée » et « grave ».

Différences statistiquement significatives entre les modalités, les individus assignés à l'OTN sans sédation ayant manifesté plus d'anxiété durant l'intervention $(p<0,01)$.

Résumé narratif : Les auteurs ont fait état de la moyenne pour l'anxiété durant l'intervention. Elle n'a pas différé entre le groupe sous OTN sans sédation et OGD transorale sans sédation $(p=0,99)$.
Très faible

Très faiblet

Très faible

Très faiblet

ran

Très faible†

Très faible†

Très faible $†$

\begin{tabular}{|l|}
\hline Très faiblet \\
\hline Très faible† \\
\hline Très faible† \\
\hline Très faible \\
\hline Très faible \\
\hline Très faible \\
\hline Très faible faible†
\end{tabular}

\footnotetext{
Remarque : $\mathrm{ERC}=$ essai randomisé et contrôlé, GRADE = Grading of Recommendations Assessment, Development and Evaluation, IC = intervalle de confiance, OGD = œsophagogastroduodénoscopie, OTN = œsophagoscopie transnasale, OVC = œsophagoscopie par vidéo capsule, $\mathrm{RGO}=$ reflux gastro-œsophagien, $\mathrm{SD}=$ écart type, $\mathrm{SE}=$ erreur type. *OTN sans sédation combinée 0/72 (OTN à l'hôpital) et 0/76 (OTN en clinique ambulatoire).

†Une évaluation GRADE de très faible certitude a été donnée parce que le risque de biais a été jugé très important. De plus le caractère indirect des données et leur imprécision ont été jugés importants.

fUne évaluation GRADE de très faible certitude a été donnée parce que le risque de biais, le caractère indirect des données et leur imprécision ont été jugés importants.
} 


\section{Valeurs et préférences des patients}

Une revue systématique des valeurs et préférences des patients ${ }^{34,35}$ n'a permis de trouver aucune donnée probante sur la façon dont les patients soupèsent les bénéfices et les préjudices associés au dépistage. Par contre, des données probantes sur les facteurs qui influent sur la décision de subir le dépistage (acceptabilité) ont été trouvées dans 3 études qui comparaient diverses stratégies de dépistage endoscopique (œsophagoscopie transnasale sans sédation ${ }^{41,45}$, œsophagoscopie par vidéocapsule [avalée] ${ }^{41}$, endoscopie perorale sans sédation ${ }^{45,49}$ et endoscopie sous sédation $)^{49}$. Dans un essai, sur les 1210 participants sollicités, $52 \%$ n'ont pas répondu à l'invitation, $32 \%$ ont refusé le dépistage (sans justification), $1 \%$ n'y étaient pas admissibles et 0,2\% ont invoqué une incapacité d'y participer ${ }^{41}$. Deux autres études ont fait état de taux de refus élevés (refus déclaré ou intentionnel) (45/105 patients, 43\%; 19/62 patients, $31 \%)$ pour les raisons suivantes : anxiété, absence d'intérêt, crainte du réflexe nauséeux, refus de participer à une étude ou réticence vis-à-vis de la voie d'insertion transnasale ${ }^{45,49}$.

Des sondages et des groupes de discussion menés par l'équipe de l'application des connaissances en vue de cette ligne directrice ont révélé qu'en considérant le rapport préjudices:bénéfices, les participants qui souffraient de RGO chronique exprimaient un intérêt modéré pour le dépistage (valeur médiane $=6$ sur 9 , sur une échelle de 1 = refus catégorique à $9=$ acceptation inconditionnelle $)^{50}$. Pour plusieurs de ces répondants, des facteurs de risque individuels ou familiaux, des convictions personnelles ou la crainte de passer à côté d'un diagnostic précoce ont eu plus de poids que les craintes associées au dépistage (p. ex., caractère effractif et complications). Pour certains autres répondants, la faible certitude des données probantes et le risque perçu associé au dépistage auraient conduit à la décision de refuser le dépistage ${ }^{50}$. À partir de cet éventail d'observations tirées des essais et des groupes de discussion, les valeurs et les préférences relatives au dépistage ont été jugées variables.

\section{Utilisation des ressources}

Étant donné la très faible certitude des données et l'absence de données probantes montrant un bénéfice associé au dépistage, aucune évaluation économique ou revue systématique sur le rapport coût-efficacité n'ont été réalisées. Les coûts potentiels du dépistage incluent les frais médicaux, les dépenses des hôpitaux/cliniques et les analyses de biopsie (11,52. $^{2}$

\section{Faisabilité, acceptabilité, coût et équité}

De l'avis du Groupe d'étude canadien, les enjeux de faisabilité et de coûts sont importants, étant donné que le RGO chronique est une maladie très répandue $(10 \%-20 \%$ de la population canadienne $)^{13,14}$. Des rapports canadiens montrent que les temps d'attente pour les endoscopies sont considérés comme trop longs et excèdent les objectifs recommandés ${ }^{53,54}$. L'application du dépistage augmenterait la demande et pourrait aggraver les problèmes d'équité en matière de santé, puisque les immigrants récents et les populations rurales ou éloignées, autochtones ou à faible revenu risqueraient de ne pas y avoir aussi facilement accès ${ }^{55,56}$. Étant donné l'incertitude des données et le manque de données probantes sur l'efficacité, nous sommes d'avis qu'il ne serait ni faisable ni acceptable de procéder au dépistage de tous les patients atteints de RGO, qui pourrait mobiliser à mauvais escient beaucoup de ressources en santé.

\section{Justification}

Globalement, le degré de certitude des données probantes était très faible. Une petite étude de cohorte rétrospective a comparé le dépistage à l'absence de dépistage et conclu que, même si les patients ayant déjà subi une œsophagogastroduodénoscopie étaient statistiquement plus susceptibles d'être atteints d'un adénocarcinome de stade moins avancé au moment du diagnostic, il n'y avait pas de différence statistiquement significative sur la survie (c.-à-d., absence de bénéfice) ${ }^{39}$. Un seul événement indésirable grave associé au dépistage a été signalé lors de 2 petits essais, qui comparaient des modalités de dépistage (très faible certitude des données probantes) ${ }^{33,35}$. Les valeurs et les préférences des patients étaient variables, puisque certains se disaient modérément intéressés par le dépistage (en fonction de leur évaluation des bénéfices et préjudices) ${ }^{50}$; toutefois, la participation réelle aux essais sur le dépistage a été faible ${ }^{34,35}$. De plus, le dépistage de tous les adultes qui souffrent de RGO chronique mobiliserait des ressources substantielles.

Certains traitements endoscopiques permettent d'éradiquer la dysplasie, mais la synthèse des revues systématiques sur ce type de données probantes indirectes a montré des degrés très variés de certitude des données probantes, allant de très faible à faible ${ }^{35,47}$. L'efficacité du traitement médical de l'œsophage de Barrett reste à confirmer.

Étant donné que nous n'avons observé qu'un stade statistiquement plus précoce au moment du diagnostic (dans une étude de cohorte rétrospective ${ }^{39}$ ), sans différence sur la survie ou d'autres issues cliniques importantes pour les patients, le Groupe d'étude canadien recommande de ne pas faire de dépistage. La recommandation est forte parce qu'en vertu de son cadre de référence pour passer des données probantes au libellé d'une décision, le Groupe d'étude canadien a accordé une valeur élevée aux ressources requises pour faire le dépistage chez tous les patients atteints de RGO à l'échelle du système, sans données probantes quant à un bénéfice (annexe 1B). Comme le mentionne une ligne directrice précédente ${ }^{36}$, lorsqu'il y a absence de données probantes pour croire qu'il y a un bénéfice à instaurer un nouveau service de prévention et qu'il y a une forte certitude que les ressources limitées du système de santé seraient dépensées, le Groupe d'étude canadien peut formuler une recommandation forte à l'encontre de l'application dudit service. Ceci concorde avec l'approche GRADE ${ }^{38}$, selon laquelle des recommandations fortes sont parfois formulées sur la base de données probantes de faible certitude, alliées à une forte certitude quant à des préjudices ou quant à une utilisation indue des ressources, compte tenu de la valeur qu'accorde le Groupe d'étude canadien à l'utilisation judicieuse des ressources en soins de santé primaires. 


\section{Méthodologie}

Le Groupe d'étude canadien sur les soins de santé préventifs est un comité indépendant de cliniciens et méthodologistes qui formule des recommandations sur des interventions cliniques de prévention primaire et secondaire (https://canadiantaskforce. $\mathrm{ca} /$ ?lang=fr). Un groupe de travail composé de 5 membres du Groupe d'étude canadien a rédigé cette ligne directrice avec le soutien scientifique de l'Agence de la santé publique du Canada.

Le cadre analytique utilisé pour la ligne directrice, y compris les questions clés, est accessible à l'annexe $1 \mathrm{~A}$. Nous avons sélectionné les issues cliniques jugées cruciales ou importantes par les patients (voir ci-dessous) ou par les membres du Groupe d'étude canadien pour orienter les revues systématiques et les recommandations. En fin de processus, 3 issues cliniques cruciales ont été retenues : mortalité de toute cause, mortalité par cancer et complications graves menaçant le pronostic vital ou médicalement significatives du dépistage; et 5 issues cliniques importantes: incidence de l'adénocarcinome œsophagien (par stade), qualité de vie, effets psychologiques, autres interventions médicales majeures et mineures, et surdiagnostic.

La recommandation de cette ligne directrice se fonde sur des revues systématiques concernant l'efficacité du dépistage ${ }^{33,35}$, sur les préférences et valeurs des patients $s^{34,35}$ et sur une synthèse des revues systématiques concernant l'efficacité des traitements ${ }^{35,47}$ que l'on peut tous consulter à l'adresse www.canadiantaskforce. ca/guidelines/published-guidelines/esophageal-adenocarcinoma/. Une analyse des résultats des groupes de discussion menés avec les patients ${ }^{50,57}$ a aussi documenté cette recommandation.

Le Centre d'analyse et de synthèse des données probantes de l'Institut de recherche de l'Hôpital d'Ottawa a procédé à 2 revues systématiques ${ }^{33-35}$ et à 1 synthèse des revues systématiques existantes ${ }^{35,47}$ entre septembre 2016 et juin 2018; des mises à jour de la recherche avant publication ont été menées en octobre ${ }^{33-35}$ et en novembre $2018^{35,47}$. Les protocoles ont été inscrits au registre PROSPERO (CRD42017049993; CRD42017050014; CRD42018084825) et sont accessibles à l'adresse www.canadiantaskforce.ca/guidelines/ published-guidelines/esophageal-adenocarcinoma/. ${ }^{31,32,58}$

Le Groupe d'étude canadien a utilisé l'approche GRADE (Grading of Recommendations, Assessment, Development and Evaluation) pour établir la certitude des données probantes et la force des recommandations (encadré no 1 1) $^{37}$. L'annexe 1B explique le cadre de l'approche GRADE qui permet de passer des données probantes au libellé de cette recommandation, que le Groupe d'étude canadien complet a révisée et approuvée.

\section{Participation des patients}

Le Groupe d'étude canadien s'est adjoint la collaboration des patients pour la préparation de la ligne directrice. Le groupe chargé de l'application des connaissances à l'Hôpital St. Michael, de Toronto ${ }^{50,57}$ a mené cette collaboration en 2 étapes pour lesquelles on a recruté 17 hommes et femmes âgés de 18 ans ou plus atteints de RGO chronique. À l'étape 1 , on a invité les participants à répondre à des questionnaires en ligne et à participer à des groupes de discussion téléphonique; ils ont fourni leur évaluation pour diverses issues cliniques du dépistage (bénéfices et préjudices). À l'étape 2, les participants ont été invités à revoir leur évaluation des issues cliniques après qu'on leur ait présenté une synthèse des données probantes provenant des revues systématiques.

\section{Revue externe et par des experts de contenu}

Des parties prenantes de l'extérieur et des experts de contenu du domaine clinique ont passé en revue les protocoles $^{31,32}$, les revues systématiques ${ }^{33,34}$, la synthèse des revues systématiques ${ }^{47}$ et l'ébauche de la ligne directrice. Les experts de contenu du domaine clinique collaborent avec les membres du groupe de travail du Groupe d'étude canadien pour les aider à résoudre des problèmes techniques et à comprendre les enjeux cliniques importants, en participant aux réunions du groupe de travail, en vérifiant l'exactitude des principaux documents clés et en effectuant la révision de la version définitive de la ligne directrice. Ces experts ne font pas partie du Groupe d'étude canadien et ne votent pas sur les recommandations.

\section{Gestion des intérêts concurrents}

Le soutien financier du Groupe d'étude canadien sur les soins de santé préventifs provient de l'Agence de la santé publique du Canada. Les vues de l'organisme subventionnaire n'ont aucunement influé sur le contenu de la recommandation. Tous les membres du Groupe d'étude canadien ont eu à dévoiler tout conflit d'intérêts d'ordre financier ou autre. Les énoncés de conflits d'intérêts sont accessibles au public sur le site Web du Groupe d'étude canadien. Un membre du Groupe d'étude canadien (H.S.) a déclaré être gastroentérologue et effectuer des endoscopies dans le cadre de sa pratique clinique; il a participé à titre de membre sans droit de vote au groupe de travail. Les autres membres de Groupe d'étude canadien ont tous déclaré ne pas être en situation de conflit d'intérêts en ce qui concerne cette ligne directrice.

Les experts de contenu du domaine clinique doivent dévoiler tout conflit d'intérêts dès le début de leur participation et annuellement par la suite. Un expert a déclaré un conflit d'intérêts en lien avec une recherche soutenue en partie par des fonds industriels. Conformément au manuel des procédures du Groupe d'étude canadien, la direction de ce dernier a révisé cette déclaration. Il a été déterminé que le conflit d'intérêts déclaré était sans lien avec la présente ligne directrice et qu'il n'aurait aucun effet prévisible sur la transparence, l'intégrité et l'acceptabilité de la ligne directrice.

\section{Mise en œuvre}

Les cliniciens devraient être à l'affût des symptômes d'alarme d'un adénocarcinome œsophagien et évaluer, orienter et prendre en charge leurs patients en conséquence. Ils devraient aussi faire preuve de jugement clinique lorsqu'il est question d'épreuves diagnostiques et de prise en charge des patients réfractaires au traitement du RGO ou présentant des symptômes évocateurs d'un autre problème des voies digestives hautes (p. ex., dyspepsie). 


\section{Suivi et évaluation}

Le degré de connaissances des cliniciens au sujet d'une recommandation est un indicateur de son adoption dans la pratique. Les taux d'endoscopie de dépistage pourraient aussi être suivis pour mesurer l'adoption de la recommandation. Le Groupe d'étude canadien suivra la publication de nouvelles données probantes et le cas échéant, mettra la recommandation à jour s'il appert qu'elles peuvent influer sur son orientation ou sa solidité.

Tableau 3 : Recommandations nationales et internationales sur le dépistage de l'adénocarcinome cesophagien, de l'œesophage de Barrett ou de la dysplasie

\section{Groupe chargé des lignes directrices}

Groupe d'étude canadien sur les soins de santé préventifs (2020)

Groupe de consensus Benign Barrett's and CAncer Taskforce «BoB CAT » ${ }^{\star}, 2015^{20}$

American College of Gastroenterology, $2015^{5}$

American Society for Gastrointestinal Endoscopy, $2015^{62}$

National Institute for Health and Care Excellence, $2014^{61}$

British Society of Gastroenterology, $2013^{4}$

American Gastroenterological Association, $2011^{60}$

Association canadienne de gastroentérologie, $2004^{59}$

\section{Recommandations}

Le Groupe d'étude canadien recommande de ne pas faire de dépistage de l'adénocarcinome œsophagien ou de ses précurseurs (œsophage de Barrett ou dysplasie) chez les adultes qui souffrent de RGO chronique (recommandation forte; très faible certitude des données probantes).

Cette ligne directrice suggère de ne pas faire de dépistage de l'œsophage de Barrett par voie endoscopique ou non endoscopique dans la population générale (recommandation conditionnelle, données probantes de faible qualité).

Le dépistage dans la population générale n'est pas recommandé (recommandation conditionnelle, données probantes de niveau faible).

Le dépistage de l'œsophage de Barrett peut être envisagé chez les hommes qui souffrent de symptômes de reflux gastro-œsophagien chronique ( $>5$ ans) ou fréquents (hebdomadaires ou plus) et qui présentent 2 facteurs de risque ou plus associés à l'œsophage de Barrett ou à l'adénocarcinome œsophagient (recommandation forte, données probantes de niveau moyen). Le dépistage de l'œsophage de Barrett chez les femmes n'est pas recommandé. Toutefois, un dépistage peut être envisagé au cas par cas selon la présence de multiples facteurs de risque associés à l'œsophage de Barrett ou à l'adénocarcinome œsophagienł (recommandation forte, données probantes de niveau faible).

Cette ligne directrice recommande l'œsophagogastroduodénoscopie chez les patients qui ont des symptômes de reflux gastro-œsophagien compliqués ou des symptômes d'alarme§ (données probantes de qualité moyenne).

La ligne directrice suggère d'envisager l'endoscopie chez les patients qui présentent plusieurs facteurs de risque associés à l'œsophage de Barrettฯ (données probantes de très faible qualité).

L'endoscopie ne devrait pas être systématiquement offerte pour le diagnostic de l'œsophage de Barrett, mais envisagée si la personne souffre de reflux gastro-œsophagien. On recommande de discuter des préférences de la personne et de ses facteurs de risque personnels (p. ex., durée prolongée ou fréquence accrue des symptômes, œsophagite antérieure, antécédents de hernie hiatale, sténose œsophagienne ou ulcères de l'œsophage, ou sexe masculin).

Le dépistage endoscopique n'est ni faisable ni justifié dans une population non sélectionnée atteinte de reflux gastro-œsophagien (recommandation de catégorie B).

Le dépistage endoscopique peut être envisagé chez les patients qui ont des symptômes de reflux gastroœsophagien chroniques et de multiples facteurs de risque (au moins 3 parmi les suivants : âge $\geq 50$ ans, race blanche, sexe masculin, obésité). Toutefois, le seuil associé aux facteurs de risque multiples doit être abaissé en présence d'antécédents familiaux (p. ex., œsophage de Barrett ou adénocarcinome œsophagien chez un parent au premier degré) (recommandation de catégorie C).

La ligne directrice recommande de ne pas faire de dépistage de l'œsophage de Barrett dans la population générale atteinte de reflux gastro-œsophagien (recommandation forte, données probantes de faible qualité). Chez les patients qui ont plusieurs facteurs de risque associés à l'adénocarcinome œsophagien (âge $\geq 50$ ans, sexe masculin, race blanche, reflux gastro-œsophagien chronique, hernie hiatale, IMC élevé et distribution intraabdominale des graisses corporelles), la ligne directrice suggère le dépistage de l'œsophage de Barrett (recommandation faible, données probantes de qualité moyenne).

Le dépistage endoscopique de l'œsophage de Barrett chez les patients qui souffrent de reflux gastroœsophagien de longue date n'a pas été associé à une réduction de la mortalité par adénocarcinome œsophagien (données probantes de niveau III, recommandation de catégorie C).

Remarque : IMC = indice de masse corporelle, $\mathrm{RGO}=$ reflux gastro-œsophagien .

*Groupe de base international pour une revue systématique des données probantes, appuyé par l'International Society of Diseases of the Esophagus, l'Esophageal Charity Fund of Ireland, Fight Esophageal Reflux Together, l'Association of Upper GI Surgeons, la British Society of Gastroenterology, la Société allemande de gastroentérologie, l'American Gastroenterology Association, l'American College of Gastroenterology, l'International Working Group for Columnar Esophagus et la European Society of Thoracic Surgeons (www.isde.net/bobcat).

†Ces facteurs de risque incluent âge $>50$ ans, race blanche, obésité centrale (circonférence de la taille $>102 \mathrm{~cm}$ ou RTH >0,9), tabagisme actif ou passé et antécédents familiaux confirmés d'œsophage de Barrett ou d'adénocarcinome œsophagien (chez un parent au premier degré).

łLes facteurs de risque incluent âge $>50$ ans, race blanche, reflux gastro-œsophagien chronique ou fréquent, obésité centrale (tour de taille $>88 \mathrm{~cm}$, RTH $>0,8$, tabagisme actuel ou passé et antécédents familiaux confirmés d'œsophage de Barrett ou d'adénocarcinome œsophagien (chez un parent au premier degré) (recommandation forte, faible niveau de données probantes).

§Symptômes d'alarme : dysphagie, odynophagie, perte de poids, saignements ou anémie.

ILes facteurs de risque incluent race blanche, sexe masculin, âge élevé ( $>50$ ans), symptômes prolongés de reflux gastro-œsophagien ( $>5$ ans), antécédents familiaux d'œsophage de Barrett ou d'adénocarcinome œsophagien, symptômes de reflux nocturne, hernie hiatale, IMC élevé ( $\geq 25 \mathrm{~kg} / \mathrm{m} 2)$, tabagisme et distribution intraabdominale des graisses. 


\section{Autres lignes directrices}

L'Association canadienne de gastroentérologie, la British Society of Gastroenterology et l'American Gastroenterological Association recommandent toutes de ne pas faire de dépistage systématique chez les patients non sélectionnés qui souffrent de RGO chronique ${ }^{4,59,60}$. Certaines lignes directrices ${ }^{4,5,60-62}$ suggèrent le dépistage chez les patients atteints de RGO qui présentent de multiples facteurs de risque (tableau 3 ). Ces recommandations ne reposent pas sur des études concernant le dépistage, mais utilisent plutôt des données épidémiologiques qui montrent une corrélation entre certains facteurs de risque spécifiques (p. ex., âge élevé [ $\geq 50$ ans], sexe masculin, obésité abdominale) et le développement d'un œsophage de Barrett ou d'un adénocarcinome œsophagien ${ }^{4-10}$. Certaines lignes directrices ont aussi intégré des études de modélisation économique ${ }^{5,62,63}$ ou l'opinion d'experts ${ }^{4}$ en plus de l'analyse des facteurs de risque. En outre, des études de cohorte sur l'œsophage de Barrett montrent que le suivi pourrait offrir un léger bénéfice sur le plan de la survie ${ }^{64-69}$. Toutefois, ce bénéfice pourrait surtout être le reflet d'un biais de devancement; nous avons exclu de notre ligne directrice les patients qui avaient déjà un diagnostic d'œsophage de Barrett.

\section{Lacunes dans les connaissances}

L'usage limité d'une définition commune du RGO chronique (p. ex., gravité, durée, traitement médicamenteux), réduit la généralisabilité des études existantes. Idéalement, il faudrait disposer d'ERC bien conçus sur les effets du dépistage par rapport à l'absence de dépistage chez les patients atteints de RGO chronique. Les obstacles à la faisabilité, toutefois, incluent la faible prévalence de l'adénocarcinome œsophagien et la probabilité limitée que les patients qui souffrent de RGO évoluent vers un cancer. On a besoin de recherches visant à évaluer des techniques de dépistage moins invasives et moins coûteuses en termes de ressources (p. ex., le dispositif Cytosponge ou d'autres dispositifs avalés) ${ }^{70,71}$ ou à clarifier l'efficacité des traitements. Par exemple, un récent ERC $(n=2557)$ a fait état d'une amélioration au fil du temps de la mortalité de toute cause, de l'adénocarcinome œsophagien ou de la dysplasie de haut grade chez des cas d'œsophage de Barrett traités par une association d'inhibiteurs de la pompe à protons à dose élevée et d'acide acétylsalicylique ${ }^{72}$. Par contre, cet ERC ne répondait pas à nos critères d'inclusion ${ }^{47}$ puisqu'il n'a pas encore été intégré à une revue systématique. Des formes plus récentes de traitement chirurgical (p. ex., combinaison de résection muqueuse endoscopique et ablation par radiofréquence, et dissection sous-muqueuse endoscopique) méritent également d'être évaluées.

\section{Conclusion}

Les données probantes passées en revue pour cette ligne directrice n'ont pas révélé de bénéfices cliniquement significatifs associés au dépistage de l'adénocarcinome œsophagien ou de ses précurseurs chez les adultes atteints de RGO chronique. Elles n'ont pas non plus fourni suffisamment de données dans les différentes catégories de facteurs de risque (p. ex., âge élevé [ $\geq 50$ ans], sexe masculin, obésité abdominale) pour appuyer la modification de notre recommandation quant au dépistage en fonction de ces facteurs, seuls ou en combinaison. Le Groupe d'étude canadien recommande fortement aux cliniciens de ne pas offrir de dépistage aux adultes qui souffrent de RGO chronique. Cette ligne directrice ne s'applique pas aux personnes qui manifestent des symptômes d'alarme ni à celles qui ont déjà un diagnostic d'œsophage de Barrett (avec ou sans dysplasie), qui devraient être évaluées, orientées et prises en charge en conséquence.

\section{Références}

1. Canadian Cancer Statistics Advisory Committee. Canadian Cancer Statistics 2019. Toronto: Canadian Cancer Society; 2019.

2. Otterstatter MC, Brierley JD, De P, et al. Esophageal cancer in Canada: trends according to morphology and anatomical location. Can J Gastroenterol 2012;26:723-7.

3. Canadian Cancer Society's Steering Committee. Canadian Cancer Statistics 2010. Toronto: Canadian Cancer Society; 2010.

4. Fitzgerald RC, di Pietro M, Ragunath K, et al. British Society of Gastroenterology guidelines on the diagnosis and management of Barrett's oesophagus. Gut 2014;63:7-42.

5. Shaheen NJ, Falk GW, lyer PG, et al.; American College of Gastroenterology. ACG clinical guideline: diagnosis and management of Barrett's esophagus. Am J Gastroenterol 2016;111:30-50.

6. Spechler SJ, Souza RF. Barrett's esophagus. N Engl J Med 2014;371:836-45.

7. Sami SS, Ragunath K, Iyer PG. Screening for Barrett's esophagus and esophageal adenocarcinoma: rationale, recent progress, challenges, and future directions. Clin Gastroenterol Hepatol 2015;13:623-34.

8. Lepage C, Drouillard A, Jouve J, et al. Epidemiology and risk factors for oesophageal adenocarcinoma. Dig Liver Dis 2013;45:625-9.

9. Rubenstein JH. Risk factors for Barrett's esophagus. Curr Opin Gastroenterol 2014;30:408-14.

10. Runge TM, Abrams JA, Shaheen NJ. Epidemiology of Barrett's esophagus and esophageal adenocarcinoma. Gastroenterol Clin North Am 2015;44:203-31.

11. Vakil N, van Zanten SV, Kahrilas P, et al.; Global Consensus Group. The Montreal definition and classification of gastroesophageal reflux disease: a global evidence-based consensus. Am J Gastroenterol 2006;101:1900-20.

12. Moayyedi PM, Lacy BE, Andrews CN, et al. ACG and CAG clinical guideline: management of dyspepsia. Am J Gastroenterol 2017;112:1484.

13. Dent J, El-Serag HB, Wallander M, et al. Epidemiology of gastro-oesophageal reflux disease: a systematic review. Gut 2005;54:710-7.

14. Fedorak RN, Veldhuyzen van Zanten S, Bridges R. Canadian Digestive Health Foundation Public Impact Series: gastroesophageal reflux disease in Canada: incidence, prevalence, and direct and indirect economic impact. Can J Gastroenterol 2010;24:431-4.

15. Rubenstein JH, Taylor J. Meta-analysis: the association of oesophageal adenocarcinoma with symptoms of gastro-oesophageal reflux. Aliment Pharmacol Ther 2010;32:1222-7.

16. Jankowski J, Barr H, Wang K, et al. Diagnosis and management of Barrett's oesophagus. BMJ 2010;341:C4551.

17. Rubenstein JH, Scheiman JM, Sadeghi S, et al. Esophageal adenocarcinoma incidence in individuals with gastroesophageal reflux: synthesis and estimates from population studies. Am J Gastroentero/ 2011;106:254-60.

18. Shaheen NJ, Weinberg D, Denberg T, et al. Upper endoscopy for gastroesophageal reflux disease: best practice advice from the clinical guidelines committee of the American College of Physicians. Ann Intern Med 2012;157:808-16.

19. Reid BJ, Li X, Galipeau PC, et al. Barrett's oesophagus and oesophageal adenocarcinoma: time for a new synthesis. Nat Rev Cancer 2010;10:87-101.

20. Bennett C, Moayyedi P, Corley DA, et al. BOB CAT: a large-scale review and Delphi Consensus for management of Barrett's esophagus with no dysplasia, indefinite for, or low-grade dysplasia. Am J Gastroenterol 2015;110:662-82.

21. McQuaid KR, Laine L, Fennerty M, et al. Systematic review: the role of bile acids in the pathogenesis of gastro-oesophageal reflux disease and related neoplasia. Aliment Pharmacol Ther 2011;34:146-65.

22. Wani S, Rubenstein JH, Vieth M, et al. Diagnosis and management of low-grade dysplasia in Barrett's esophagus: expert review from the Clinical Practice Updates Committee of the American Gastroenterological Association. Gastroenterology 2016;151:822-35 
23. Peters CJ, Fitzgerald R. Systematic review: the application of molecular pathogenesis to prevention and treatment of oesophageal adenocarcinoma. Aliment Pharmacol Ther 2007;25:1253-69.

24. Desai TK, Krishnan K, Samala N, et al. The incidence of oesophageal adenocarcinoma in non-dysplastic Barrett's oesophagus: a meta-analysis. Gut 2012;61:970-6.

25. Spechler SJ. Barrett esophagus and risk of esophageal cancer: a clinical review. JAMA 2013;310:627-36.

26. Singh S, Manickam P, Amin A, et al. Incidence of esophageal adenocarcinoma in Barrett's esophagus with low-grade dysplasia: a systematic review and meta-analysis. Gastrointest Endosc 2014;79:897-909.e4.

27. Hippisley-Cox J, Coupland C. Identifying patients with suspected gastrooesophageal cancer in primary care: derivation and validation of an algorithm. Br J Gen Pract 2011;61:e707-14.

28. Canadian Cancer Statistics Advisory Committee. Canadian Cancer Statistics 2018. Toronto: Canadian Cancer Society; 2018.

29. Vaughan TL, Fitzgerald RC. Precision prevention of oesophageal adenocarcinoma. Nat Rev Gastroenterol Hepatol 2015;12:243-8.

30. Atun R. What are the advantages and disadvantages of restructuring a health care system to be more focused on primary care services? Copenhagen: World Health Organization Regional Office for Europe; 2004. Accessible ici : www.euro.who. int/_data/assets/pdf_file/0004/74704/E82997.pdf (consulté le 7 septembre 2018).

31. Hamel C, Beck A, Stevens A, et al. Effectiveness of screening for esophageal adenocarcinoma and precancerous conditions (dysplasia and Barrett's esophagus) in patients with chronic gastroesophageal reflux disease with or without other risk factors: protocol for a systematic review. PROSPERO 2017 CRD42017049993. 2017; Accessible ici : www.crd.york.ac.uk/prospero/display _record.php?RecordID=49993 and http://canadiantaskforce.ca/guidelines/ published-guidelines/esophageal-adenocarcinoma/ (consulté le 4 décembre 2017).

32. Hamel C, Beck A, Stevens A, et al. Patient values and preferences in relation to screening for esophageal adenocarcinoma and precancerous conditions (dysplasia and Barrett's esophagus): protocol for a systematic review. 2017; Accessible ici : www.crd.york.ac.uk/prospero/display_record.php?RecordID $=50014$ and http://canadiantaskforce.ca/guidelines/published-guidelines/ esophageal-adenocarcinoma/ (consulté le 4 décembre 2017).

33. Hamel C, Beck A, Thuku M, et al. Screening for esophageal adenocarcinoma and precancerous conditions (dysplasia and Barrett's esophagus) in patients with chronic gastroesophageal reflux disease with or without other risk factors: systematic review. Ottawa: Ottawa Hospital Research Institute; 2018.

34. Hamel C, Beck A, Stevens A, et al. Patient values and preferences in relation to screening for esophageal adenocarcinoma and precancerous conditions (dysplasia and Barrett's esophagus) in patients with chronic gastroesophageal reflux disease with or without other risk factors: Systematic review. Ottawa, Ontario: Ottawa Hospital Research Institute; 2018.

35. Hamel C, Ahmadzai N, Beck A, et al. Screening for esophageal adenocarcinoma and precancerous conditions (dysplasia and Barrett's esophagus) in patients with chronic gastroesophageal reflux disease with or without other risk factors: two systematic reviews and one overview of reviews to inform a guideline of the Canadian Task Force on Preventive Health Care (CTFPHC). Syst Rev 2020;9; doi:10.1186/s13643-020-1275-2.

36. Klarenbach S, Sims-Jones N, Lewin G, et al. Recommendations on screening for breast cancer in women aged 40-74 years who are not at increased risk for breast cancer. CMAJ 2018;190:E1441-51.

37. Schünemann H, Brożek J, Guyatt G, et al. GRADE handbook: handbook for grading the quality of evidence and strength of recommendations using the GRADE approach. GRADE Working Group; 2013. Accessible ici : http://gdt. guidelinedevelopment.org/app/handbook/handbook.html (consulté le 4 décembre 2017).

38. Andrews JC, Schünemann HJ, Oxman AD, et al. GRADE guidelines: 15. Going from evidence to recommendation-determinants of a recommendation's direction and strength. J Clin Epidemiol 2013;66:726-35.

39. Rubenstein JH, Sonnenberg A, Davis J, et al. Effect of a prior endoscopy on outcomes of esophageal adenocarcinoma among United States veterans. Gastrointest Endosc 2008;68:849-55

40. Hammad TA, Thrift AP, El-Serag HB, et al. Missed opportunities for screening and surveillance of Barrett's esophagus in veterans with esophageal adenocarcinoma. Dig Dis Sci 2019;64:367-72.

41. Chak A, Alashkar BM, Isenberg GA, et al. Comparative acceptability of transnasal esophagoscopy and esophageal capsule esophagoscopy: a randomized, controlled trial in veterans. Gastrointest Endosc 2014;80:774-82.

42. Chang JY, Talley NJ, Locke GR III, et al. Population screening for Barrett esophagus: a prospective randomized pilot study. Mayo Clin Proc 2011;86:1174-80.
43. Sami SS, Dunagan KT, Johnson ML, et al. A randomized comparative effectiveness trial of novel endoscopic techniques and approaches for Barrett's esophagus screening in the community. Am J Gastroenterol 2015;110:148-58.

44. Jobe BA, Hunter JG, Chang EY, et al. Office-based unsedated small-caliber endoscopy is equivalent to conventional sedated endoscopy in screening and surveillance for Barrett's esophagus: a randomized and blinded comparison. Am J Gastroenterol 2006;101:2693-703.

45. Zaman A, Hahn M, Hapke R, et al. A randomized trial of peroral versus transnasal unsedated endoscopy using an ultrathin videoendoscope. Gastrointest Endosc 1999;49:279-84.

46. Mori A, Ohashi N, Yoshida A, et al. Unsedated transnasal ultrathin esophagogastroduodenoscopy may provide better diagnostic performance in gastroesophageal reflux disease. Dis Esophagus 2011;24:92-8.

47. Ahmadzai N, Hamel C, Thuku M, et al. Benefits and harms of treatment options for esophageal adenocarcinoma and precancerous conditions: an overview of systematic reviews. Ottawa: Ottawa Hospital Research Institute; 2018.

48. Lordick F, Mariette C, Haustermans K, et al.; ESMO Guidelines Committee. Oesophageal cancer: ESMO Clinical Practice Guidelines for diagnosis, treatment and follow-up. Ann Oncol 2016;27(Suppl 5):v50-v57.

49. Zaman A, Hapke R, Sahagun G, et al. Unsedated peroral endoscopy with a video ultrathin endoscope: patient acceptance, tolerance, and diagnostic accuracy. Am J Gastroenterol 1998;93:1260-3.

50. Buckland D, Sayal R, Fredrickson K, et al. Patient preferences for esophageal adenocarcinoma screening: data summary. Prepared for the Canadian Task Force on Preventive Health Care. Toronto: Knowledge Translation Program, Li Ka Shing Knowledge Institute, St. Michael's Hospital; 2018.

51. Roth LS, Adams PC. Variation in physician reimbursement for endoscopy across Canada. Can J Gastroenterol 2009;23:503-5.

52. Crott R, Makris N, Barkun A, et al. The cost of an upper gastroduodenal endoscopy: an activity-based approach. Can J Gastroenterol 2002;16:473-82.

53. Paterson WG, Barkun AN, Hopman WM, et al. Wait times for gastroenterology consultation in Canada: the patients' perspective. Can J Gastroentero/ 2010;24: 28-32.

54. Leddin D, Armstrong D, Borgaonkar M, et al. The 2012 SAGE wait times program: Survey of Access to GastroEnterology in Canada. Can J Gastroenterol 2013;27:83-9

55. Buchman S, Rozmovits L, Glazier R. Equity and practice issues in colorectal cancer screening. Can Fam Physician 2016;62:e186-e193.

56. Ahmed S, Shahid R, Episkenew J. Disparity in cancer prevention and screening in aboriginal populations: recommendations for action. Curr Oncol 2015; 22:417-26.

57. Buckland D, Sayal BN, Moore J, et al. Patient preferences in considering esophageal cancer screening outcomes: prepared for the Canadian Task Force on Preventive Health Care. Toronto: Knowledge Translation Program, Li Ka Shing Knowledge Institute, St. Michael's Hospital; 2016.

58. Hamel C, Groulx S, Doull M, et al. Benefits and harms of treatment options for esophageal adenocarcinoma and precancerous conditions: a protocol for an overview of systematic reviews. PROSPERO 2018 CRD42018084825. London (UK): National Institute for Health Research; 2018. Accessible ici : www.crd. york.ac.uk/PROSPERO/display_record.php?ID=CRD42018084825 (consulté le 4 décembre 2017).

59. Armstrong D, Marshall JK, Chiba N, et al. Canadian Consensus Conference on the management of gastroesophageal reflux disease in adults - update 2004 . Can J Gastroenterol 2005;19:15-35.

60. Spechler SJ, Sharma P, Souza RF, et al. American Gastroenterological Association medical position statement on the management of Barrett's esophagus. Gastroenterology 2011;140:1084-91.

61. National Institute for Health and Care Excellence (NICE). Dyspepsia and gastrooesophageal reflux disease in adults: quality standard. London (UK): NICE; 2014. Accessible ici : www.nice.org.uk/guidance/qs96/resources/dyspepsia -and-gastrooesophageal-reflux-disease-in-adults-pdf-2098972399813 (consulté le 20 juin 2017).

62. ASGE Standards of Practice Committee; Muthusamy VR, Lightdale JR, Acosta $\mathrm{RD}$, et al. The role of endoscopy in the management of GERD. Gastrointest Endosc 2015;81:1305-10.

63. Whiteman DC, Appleyard M, Bahin FF, et al. Australian clinical practice guidelines for the diagnosis and management of Barrett's esophagus and early esophageal adenocarcinoma. J Gastroenterol Hepatol 2015;30:804-20.

64. Codipilly DC, Chandar AK, Singh S, et al. The effect of endoscopic surveillance in patients with Barrett's esophagus: a systematic review and meta-analysis. Gastroenterology 2018;154:2068-86. 
65. Wenker TN, Tan MC, Liu Y, et al. Prior diagnosis of Barrett's esophagus is infrequent, but associated with improved esophageal adenocarcinoma survival. Dig Dis Sci 2018;63:3112-9.

66. Fountoulakis A, Zafirellis KD, Dolan K, et al. Effect of surveillance of Barrett's oesophagus on the clinical outcome of oesophageal cancer. Br J Surg 2004;91: 997-1003.

67. Verbeek RE, Leenders M, Ten Kate FJW, et al. Surveillance of Barrett's esophagus and mortality from esophageal adenocarcinoma: a population-based cohort study. Am J Gastroenterol 2014;109:1215-22.

68. Kastelein F, van Olphen S, Steyerberg EW, et al. Surveillance in patients with long-segment Barrett's oesophagus: a cost-effectiveness analysis. Gut 2015; 64:864-71.
69. Tramontano AC, Sheehan DF, Yeh JM, et al. The impact of a prior diagnosis of Barrett's esophagus on esophageal adenocarcinoma survival. Am J Gastroenterol 2017;112:1256-64.

70. Offman J, Muldrew B, O'Donovan M, et al. Barrett's oESophagus trial 3 (BEST3): study protocol for a randomised controlled trial comparing the CytospongeTFF3 test with usual care to facilitate the diagnosis of oesophageal pre-cancer in primary care patients with chronic acid reflux. BMC Cancer 2018;18:784.

71. Moinova HR, LaFramboise T, Lutterbaugh JD, et al. Identifying DNA methylation biomarkers for non-endoscopic detection of Barrett's esophagus. Sci Transl Med 2018 Jan 17;10. pii: eaao5848. doi: 10.1126/scitranslmed.aao5848.

72. Jankowski JAZ, de Caestecker J, Love SB, et al. Esomeprazole and aspirin in Barrett's oesophagus (AspECT): a randomised factorial trial. Lancet 2018;392:400-8.
Auteurs : Stéphane Groulx M.D., Heather Limburg M.Sc., Marion Doull Ph.D., Scott Klarenbach M.D. M.Sc., Harminder Singh M.D. M.S.P., Brenda J. Wilson MBChB M.Sc. MRCP, Brett Thombs Ph.D; pour le Groupe d'étude canadien sur les soins de santé préventifs

Intérêts concurrents : Harminder Singh a déclaré être gastroentérologue et effectuer des endoscopies dans le cadre de sa pratique clinique. Le $D^{r}$ Singh signale aussi avoir reçu des honoraires personnels de Takeda Canada, Pendopharm et Amgen, à l'extérieur des travaux soumis. Aucun autre intérêt concurrent n'a été déclaré.

Cet article a été révisé par des pairs.

Affiliations : Département de sciences de la santé communautaire (Groulx), Université de Sherbrooke, Sherbrooke, Qué; Agence de la santé publique du Canada (Limburg, Doull), Ottawa, Ont.; Département de médecine (Klarenbach), Université de l'Alberta, Edmonton, Alta.; Médecine interne et sciences de la santé communautaire (Singh), Université du Manitoba, Winnipeg, Man.; Santé communautaire et humanités (Wilson), Université Memorial, St. John's, T.-N.-L.; Département de psychiatrie (Thombs), Hôpital général juif et Université McGill, Montréal, Qué.

Comité de rédaction de la ligne directrice : Stéphane Groulx (membre du Groupe d'étude canadien ayant droit de vote), Heather Limburg (membre de l'équipe scientifique sans droit de vote), Marion Doull (membre de l'équipe scientifique sans droit de vote), Scott Klarenbach (membre du Groupe d'étude canadien ayant droit de vote), Harminder Singh (membre du Groupe d'étude canadien sans droit de vote), Brenda J. Wilson (membre du Groupe d'étude canadien ayant droit de vote), Brett Thombs (membre du Groupe d'étude canadien ayant droit de vote)

Membres collaborateurs du Groupe d'étude canadien sur les soins de santé préventifs : Ahmed Abou-Setta, Heather Colquhoun, Roland Grad, Michael Kidd, Christina Korownyk, Eddy Lang, John C. LeBlanc, Gabriela A. Lewin (ex-membre), Ainsley Moore, Nav Persaud, Donna L. Reynolds, John J. Riva, Guylène Thériault

Collaborateurs : Stéphane Groulx, Heather Limburg, Marion Doull, Scott Klarenbach, Harminder Singh, Brenda Wilson et Brett Thombs ont contribué substantiellement à la conception et à l'élaboration de l'étude, à l'interprétation des données probantes et à la révision critique de l'ébauche de la ligne directrice. Heather Limburg et Marion Doull ont fourni un soutien scientifique et logistique au comité de rédaction. Stéphane Groulx, Scott Klarenbach, Harminder Singh, Brenda Wilson et Brett Thombs ont rédigé l'ébauche des recommandations. Stéphane Groulx, Heather Limburg et Marion Doull ont rédigé le libellé de la ligne directrice. Tous les auteurs cités ont donné leur approbation finale à la version de la ligne directrice destinée à être publiée et se portent garants de tous les aspects du travail. Tous les membres collaborateurs du Groupe d'étude canadien sur les soins de santé préventifs (Ahmed Abou-Setta, Heather Colquhoun, Roland Grad, Michael Kidd, Christina Korownyk, Eddy Lang, John LeBlanc, Gabriela Lewin [ex-membre], Ainsley Moore, Nav Persaud, Donna L. Reynolds, John Riva et Guylène Thériault) ont aussi contribué à la préparation de la ligne directrice et ont donné leur approbation finale pour la version de la ligne directrice devant être publiée.

Financement : Le financement du Groupe d'étude canadien sur les soins de santé préventifs est fourni par l'Agence de la santé publique du Canada. Les vues de l'organisme subventionnaire n'entrent pas en ligne de compte lorsque le Groupe d'étude canadien prépare ses recommandations. Les vues exprimées aux présentes n'engagent que le Groupe d'étude canadien et ne représentent pas nécessairement celles de l'Agence de la santé publique du Canada.

Remerciements : Les auteurs remercient l'équipe du Centre d'analyse et de synthèse des données de l'Institut de recherche de l'Hôpital d'Ottawa (IRHO) (Candyce Hamel, Andrew Beck, Nadera Ahmadzai, Micere Thuku, Kusala Pussegoda, Adrienne Stevens, Becky Skidmore, Avijit Chatterjee, Donna E. Maziak, Kristopher Dennis, Lise Bjerre, Lorenzo Ferri, Beverley Shea, Brian Hutton, Julian Little et David Moher) pour les revues des données probantes qui sous-tendent cette ligne directrice; Marc Avey, Susan Courage, Bradley Mitchelmore et Rachel Rodin de la Division des lignes directrices et de la santé mondiale de l'Agence de la santé publique du Canada, qui ont appuyé la préparation de la ligne directrice; et le Programme d'application des connaissances de l'Institut du savoir Li Ka Shing de l'Hôpital St. Michael pour sa contribution à l'engagement des patients et à l'application des connaissances en lien avec cette ligne directrice. Ils remercient aussi Paul J. Belletrutti, de l'Université de Calgary, Calgary, Alta., et Laura Targownik, de l'Université du Manitoba, Winnipeg, Man., pour leur aide en tant qu'experts de contenu du domaine clinique pendant la préparation de cette ligne directrice. Les experts de contenu du domaine clinique qui ont aidé le Groupe d'étude canadien durant le processus d'élaboration de la ligne directrice peuvent être d'accord ou non avec les recommandations du Groupe d'étude canadien. Les auteurs sont aussi reconnaissants envers les pairs-réviseurs et les parties prenantes organisationnelles qui ont formulé des commentaires sur l'ébauche de la ligne directrice, notamment David Armstrong, Université McMaster, Hamilton, Ont.; Shawn Chirrey, Société canadienne du cancer, Toronto, Ont.; Bruce Greenwald, Faculté de médecine de l'Université du Maryland, Baltimore (Md.); Milli Gupta, Université de Calgary, Calgary, Alta.; Prasad G. Iyer, Clinique Mayo, Rochester (Minn.); Edward Kucharski, Université de Toronto, Toronto, Ont.; Grigorios Leontiadis, Université McMaster, Hamilton, Ont., et Gilles Plourde, Santé Canada, Ottawa, Ont.

Correspondance : Groupe d'étude canadien sur les soins de santé préventifs, info@canadiantaskforce.ca 